\title{
Meta-Analysis of the Relationship between Abdominal Obesity and Diabetic Kidney Disease in Type 2 Diabetic Patients
}

\author{
Qinying Zhao ${ }^{\mathrm{a}}$ Xiaoyan $\mathrm{Yi}^{\mathrm{b}}$ Zhihong Wang ${ }^{\mathrm{a}}$ \\ a Department of Endocrinology, The First Affiliated Hospital of Chongqing Medical University, Chongqing, China; \\ ${ }^{b}$ First Branch of the First Affiliated Hospital of Chongqing Medical University, Chongqing, China
}

\section{Keywords}

Abdominal obesity · Diabetic kidney disease · Type 2 diabetes · Meta-analysis

\begin{abstract}
Background and Objectives: The meta-analysis aimed to investigate the association of visceral fat area (VFA), waist circumference (WC), waist-hip ratio (WHR) and waist-height ratio (WHtR) with diabetic kidney disease (DKD) in type 2 diabetic patients. Methods: Included studies were searched from Pubmed, Embase, and the Cochrane Library before July 2020. We synthesized the pooled results of the above relationships by meta-analysis. Results: Fourteen cross-sectional studies were enrolled. The pooled results indicated there was a significant difference in continuous VFA, WC and WHR/ WHtR between patients with DKD and those without DKD (standard mean difference, SMD, 0.24, 95\% confidence interval, $\mathrm{Cl}, 0.13-0.36, p=0.000)$. For VFA, patients with DKD had higher VFA levels than those without DKD (SMD 0.27, 95\% Cl 0.03-0.50). In the WC subgroup, patients with DKD had higher WC levels than those without DKD (SMD 0.17, 95\% CI 0.100.24); similarly, abdominal obesity (dichotomized WC) was significantly associated with an increase in the odds of DKD (expected shortfall, ES, 1.57, 95\% Cl 1.32-1.86). However, the association of continuous WHR/WHtR with DKD was not sta-
\end{abstract}

tistically significant (SMD $0.43,95 \% \mathrm{Cl}-0.12$ to 0.97 ), while we found this relationship was statistically significant when analyzed categorically (ES 1.58, 95\% Cl 1.22-2.06). Conclusion: In this meta-analysis, we found abdominal obesity parameters (continuous VFA, WC) were associated with increased odds of DKD, and type 2 diabetic patients with DKD were more likely to have abdominal obesity (categorized using WC or WHR/WHtR).

(C) 2021 The Author(s)

Published by S. Karger AG, Basel

\section{Introduction}

Diabetic kidney disease (DKD), a serious microvascular complication of diabetes, is defined as a decrease in estimated glomerular filtration rate (eGFR) or the occurrence of albuminuria. It is well known that DKD already becomes the major cause of end-stage renal disease in the world [1], which even aggravates the occurrence and development of cardiovascular disease and mortality [2]. Therefore, it is important to identify the related risk factors of DKD.

Obesity is a critical risk factor for type 2 diabetes mellitus (T2DM) and hypertension [3, 4], which is closely related to the development of DKD [5]. General and abdominal obesity are the major subtypes of obesity. Some

Correspondence to:

Zhihong Wang, towzh713@126.com

karger@karger.com www.karger.com/ofa

Karger $\stackrel{\text { ' }}{5}$

GOPEN ACCESS
(C) 2021 The Author(s)

Published by S. Karger AG, Basel

This is an Open Access article licensed under the Creative Commons Attribution-NonCommercial-4.0 International License (CC BY-NC) (http://www.karger.com/Services/OpenAccessLicense), applicable to the online version of the article only. Usage and distribution for commercial purposes requires written permission. 
studies reported that, compared with general obesity, abdominal obesity had been shown to be superior to contribute to the risk of DKD in T2DM [6-10], while Man et al. [11] found that individuals with abdominal obesity had no association with DKD in T2DM. Kanakamani et al. [12] also observed that waist circumference (WC) was not associated with microalbuminuria in 670 patients attending the endocrine outpatient clinic. It is necessary to further ascertain the relationship between abdominal obesity and DKD in type 2 diabetic patients.

In this meta-analysis, we aimed to investigate the association of visceral fat area (VFA), WC and waist-hip ratio (WHR)/waist-height ratio (WHtR) with $\mathrm{DKD}$ in type 2 diabetic patients through existing research.

\section{Methods}

\section{Assessment of Abdominal Obesity and DKD}

VFA, WC, WHR and WHtR were common markers to evaluate abdominal obesity. Abdominal obesity was defined as $\mathrm{WC}>90 \mathrm{~cm}$ for males or $80 \mathrm{~cm}$ for females [13], or WC $>102 \mathrm{~cm}$ for males and $88 \mathrm{~cm}$ for females [14], or VFA $\geq 100 \mathrm{~cm}^{2}$ [15]. DKD was defined as $\mathrm{eGFR}<60 \mathrm{~mL} / \mathrm{min} / 1.73 \mathrm{~m}^{2}$ [16], or urinary albumin excretion rate $>30 \mathrm{mg} / 24 \mathrm{~h}$ (20 mg/min) [17], or microalbuminuria (urinary albumin creatinine ratio within $30-300 \mathrm{mg} / \mathrm{g}$ creatine), or macroalbuminuria (urinary albumin creatinine ratio $>300 \mathrm{mg} / \mathrm{g}$ creatine) [1]

\section{Study Selection}

We searched Pubmed, Embase and Cochrane databases before July 2020, using the following terms: visceral fat, waist, abdominal obesity, visceral obesity, central obesity, diabetic nephropathy, diabetic kidney disease, glomerular filtration rate, eGFR, albuminuria and diabetic patients. Eligible studies met the following criteria: (1) DKD and controls were included and defined; (2) the associations of VFA, WC, WHR and WHtR with DKD were evaluated; (3) reported the standard mean difference (SMD) or odds ratio (OR) and its $95 \%$ confidence interval (CI). In these studies, we excluded duplicated studies, literature reviews, unavailable studies, animal experiments and case reports.

\section{Data Extraction}

Two independent reviewers conducted a literature search, study selection, data extraction and quality assessment. The author's name, publication year, study design, type of diabetes, marker of abdominal obesity, definition of DKD, the number of patients, the mean \pm SD of marker, adjusted OR and 95\% CI were included in each study. And the disagreement was resolved by consensus with a third reviewer.

Statistical Analysis and Quality Assessment

The meta-analysis was performed using the Stata 16.0 (Stata Corp., College Station, TX, USA). For continuous variables, which were presented as mean $\pm \mathrm{SD}$, SMD with $95 \%$ CI were calculated. While OR and 95\% CI were calculated on pooled effects for categorized variables. Heterogeneity among studies was examined by the $I^{2}$ statistic $[18,19] . I^{2}$ values of $25-50 \%, 50-75 \%$ and $75 \%$ were

Abdominal Obesity Correlates with DKD

More Closely considered indicative of low, moderate and high heterogeneity, respectively [19]. If $I^{2}>50 \%$, a random-effect model was performed to synthesize study effects; otherwise, a fixed-effect model was used. In addition, sensitivity analyses were attempted to identify potential sources of the heterogeneity. Egger's test [20] was used to evaluate publication bias, and $p<0.05$ was statistically significant. Quality assessment was evaluated using the Healthcare Research and Quality [21]. Finally, this meta-analysis complied with the Preferred Reporting Items for Systematic Reviews and Meta-Analyses (PRISMA) guidelines (shown in online suppl. Table 1; for all online suppl. material, see www.karger.com/ doi/10.1159/000516391).

\section{Results}

We initially retrieved 1,064 publications from databases and 9 citations from reference lists; of these, 29 articles were included after reading the title and abstract. After full-text review, 14 studies [9-12, 17, 22-30] involving 13,743 patients were identified and enrolled in this metaanalysis (Fig. 1). Detailed information of another $15 \mathrm{ex}-$ cluded articles is accessible in Figure 1. The baseline characteristics of these enrolled studies are shown in Table 1.

\section{DKD and All Abdominal Obesity Parameters}

A total of 14 articles were included in this meta-analysis of all abdominal obesity parameters including VFA $[17,22,23]$, WC $[10-12,22,24-26]$ and WHR/WHtR $[10,11]$ with DKD (Fig. 2). The $p$ value of heterogeneity between these studies was significant $\left(I^{2} 85.3 \%\right)$, so we used the random-effect model indicating that there was a statistically significant difference between patients with DKD and those without DKD (SMD 0.24, 95\% CI 0.13$0.36, p=0.000)$. No publication bias was found by Egger's test $(p=0.449$; shown in online suppl. Fig. S1A). We used sensitivity analysis and subgroup analysis to explore the resources of heterogeneity. The sensitivity analysis was performed to recalculate the pooled risk estimates for the remaining studies by excluding one study at a time, which resulted in little change in the observed risk estimates from 0.19 (95\% CI 0.15-0.23) to 0.25 (95\% CI 0.20-0.29; online suppl. Fig. S2A). In addition, the results of subgroup analysis by VFA, WC or WHR/WHtR were summarized as follows.

\section{$D K D$ and $V F A$}

In the VFA subgroup [17, 22, 23], we found a significant association between increased VFA and likelihood of DKD (SMD 0.27, 95\% CI 0.03-0.50, $p=0.014$; Fig. 3). High heterogeneity was observed $\left(I^{2} 76.5 \%\right)$. No publication bias was found by Egger's test $(p=0.948$; online 
Fig. 1. Flow chart of literature search and study selection.

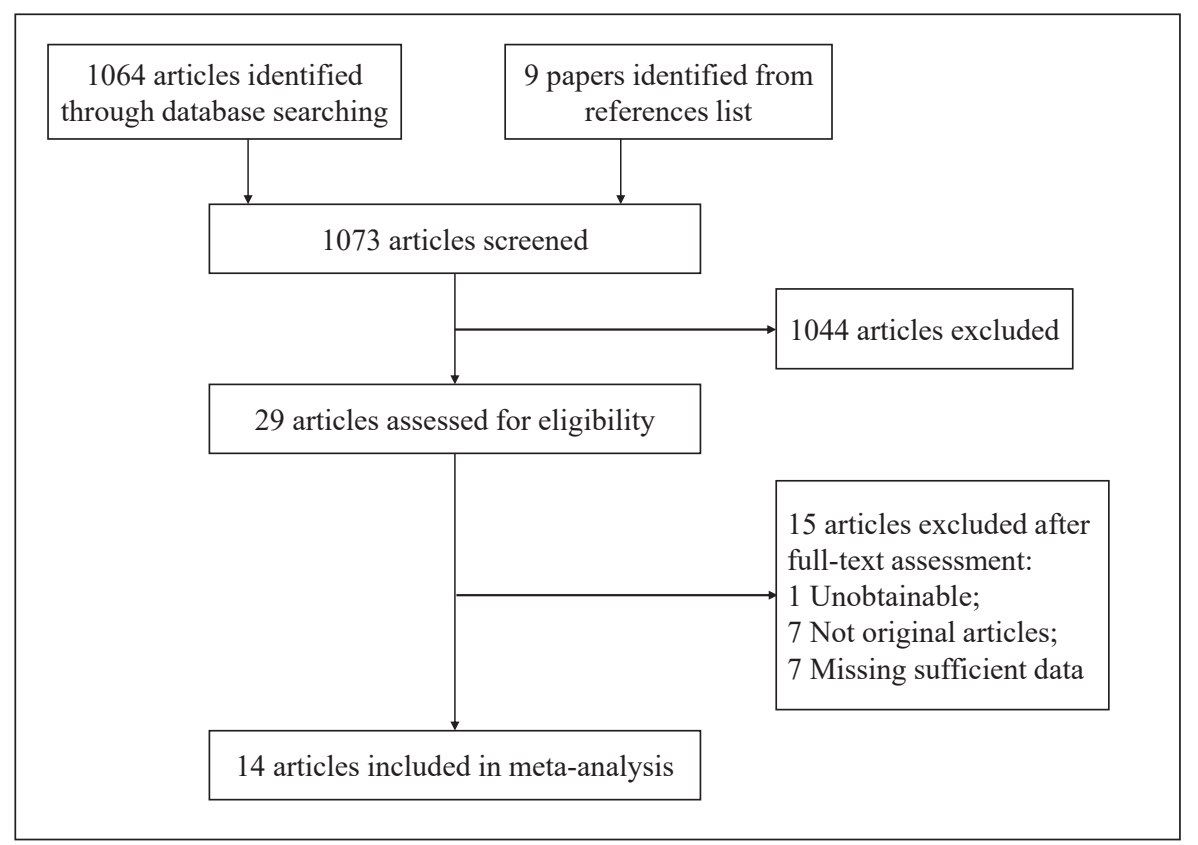

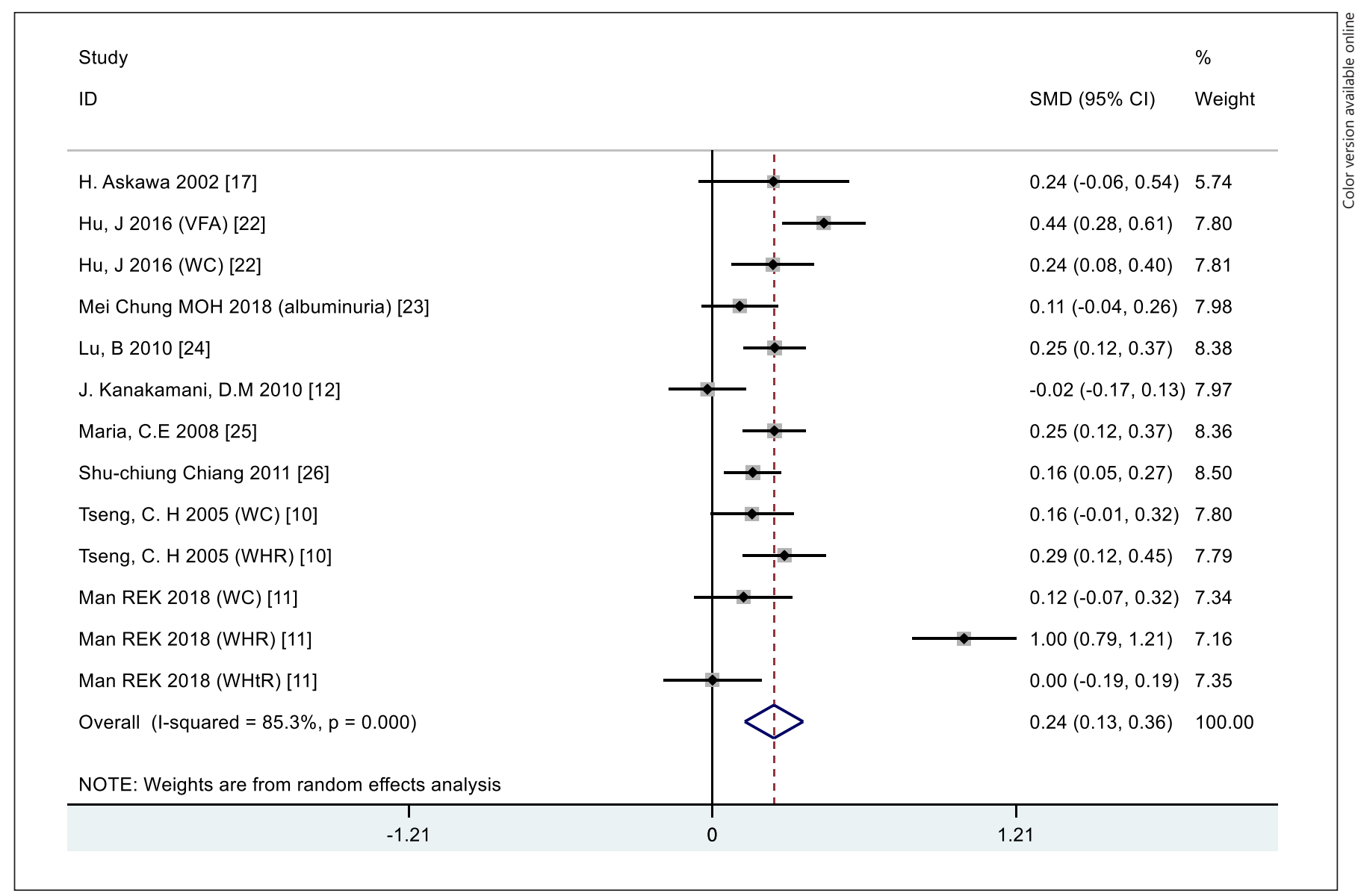

Fig. 2. Forest plot of the association of continuous waist circumference, visceral fat area and waist-hip ratio/waistheight ratio with diabetic kidney disease. 


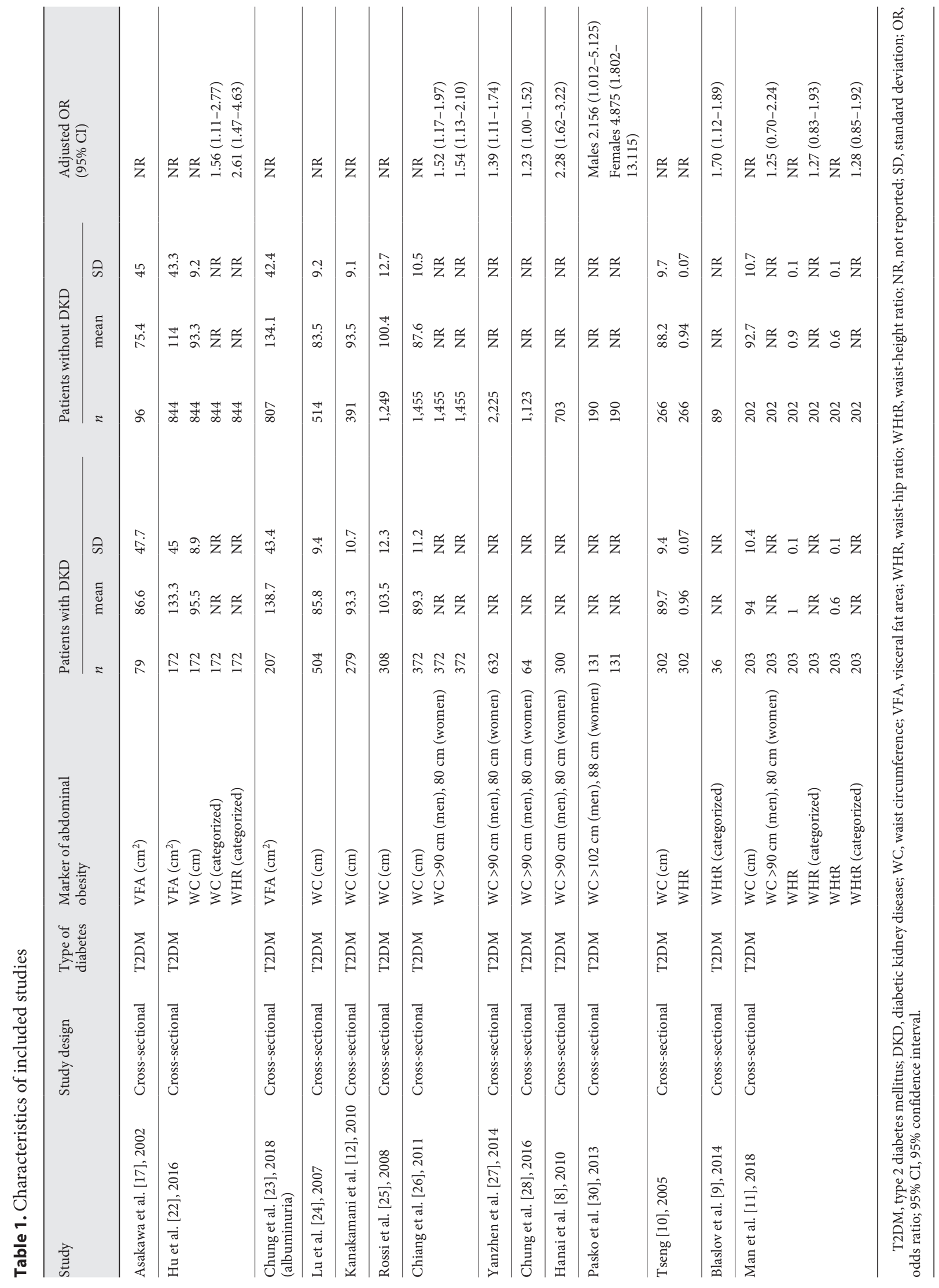


Hu, J 2016 (VFA) [22]

Mei Chung MOH 2018 (albuminuria) [23]

Overall $($ I-squared $=76.5 \%, p=0.014)$

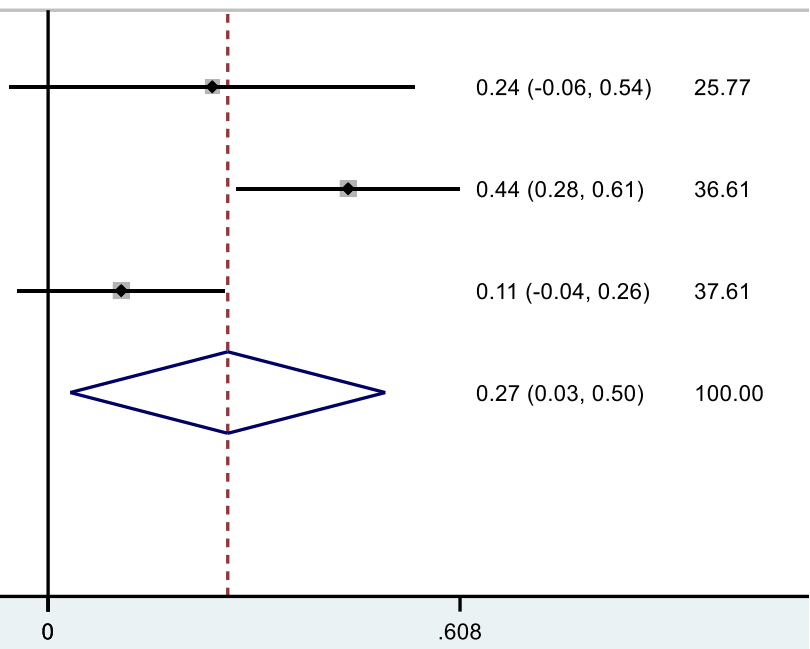

NOTE: Weights are from random effects analysis

Fig. 3. Forest plot of the association of continuous visceral fat area with diabetic kidney disease.

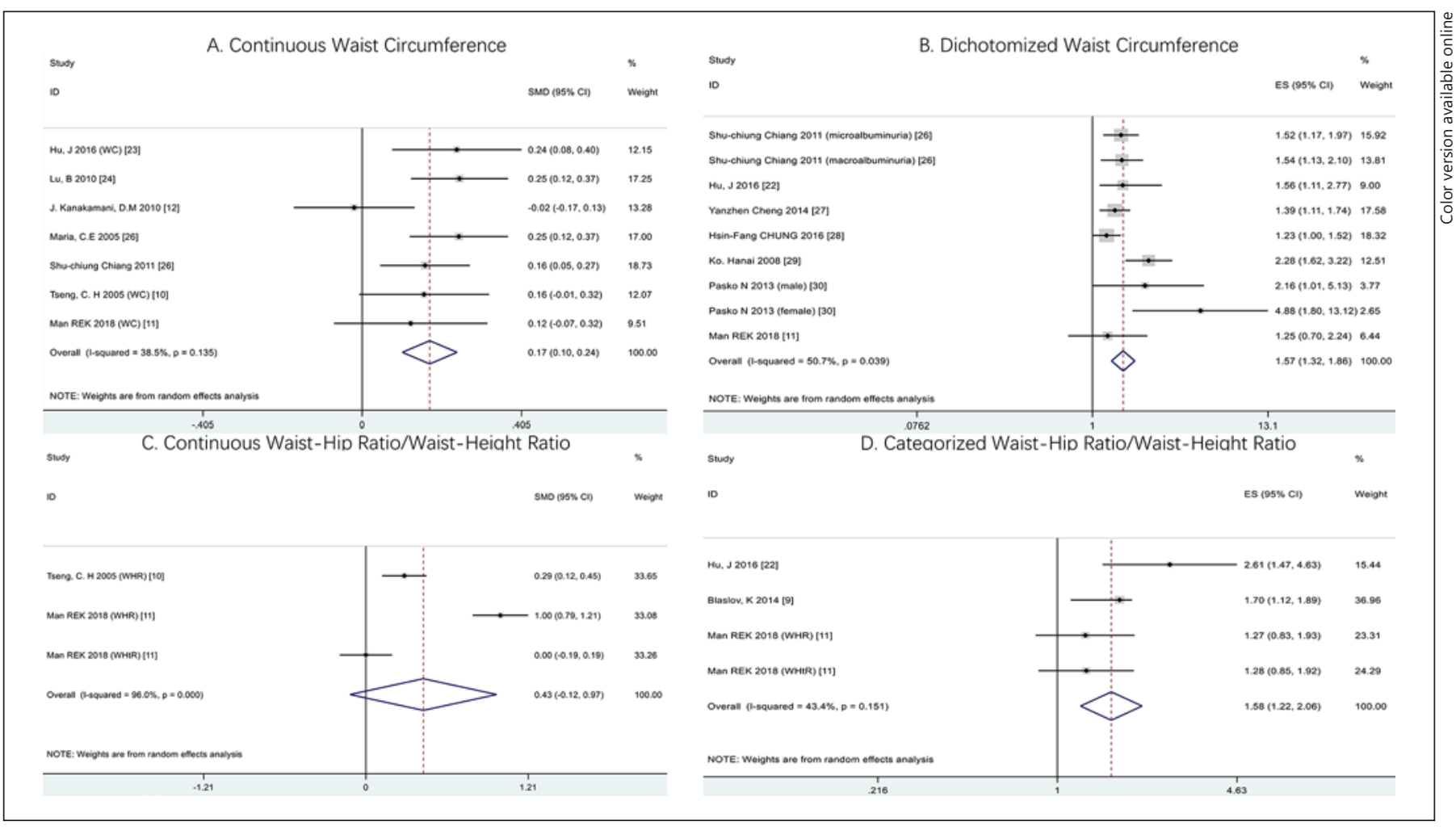

Fig. 4. Forest plot of the association of different parameters of abdominal obesity with diabetic kidney disease. 
suppl. Fig. S1B). Sensitivity analysis demonstrated that the observed risk estimate was not robust, which changed from 0.14 (95\% CI -0.00 to 0.27 ) to 0.40 (95\% CI 0.25 0.54; online suppl. Fig. S2B).

\section{$D K D$ and $W C$}

In the WC subgroup, there were 7 and 7 studies included in the meta-analysis of the effects of continuous WC [10-12, 22, 24-26] and abdominal obesity (dichotomized WC) [11, 26-30]. Patients with DKD had higher WC levels than those without DKD (SMD 0.17, 95\% CI $0.10-0.24, I^{2} 38.5 \%, p=0.135$; Fig. 4a). Egger's test showed that no publication bias existed in the meta-analysis ( $p=$ 0.439; online suppl. Fig. S3A). The sensitivity analysis presented a robust result that was not influenced by individual studies (shown in online suppl. Fig. S4A). Similarly, abdominal obesity (dichotomized WC) was significantly associated with an increase in the odds of DKD (ES 1.57, 95\% CI 1.32-1.86, $I^{2}$ 50.7\%, $p=0.039$; Fig. 4b). No publication bias existed in the subgroup analysis $(p=$ 0.061 ; online suppl. Fig. S3B). The sensitivity analysis also presented a robust result that was not influenced by individual studies (online suppl. Fig. S4B).

\section{$D K D$ and $W H R / W H t R$}

In the WHR/WHtR subgroup, 3 studies were included in the meta-analysis of the effects of continuous $[10,11]$ and categorized WHR/WHtR $[9,11]$. The association of continuous WHR/WHtR with DKD was not statistically significant (SMD 0.43, 95\% CI -0.12 to $0.97, I^{2} 96.0 \%$, $p=0.000$; Fig. 4c). No publication bias was found by Egger's test ( $p=0.686$; online suppl. Fig. S3C), but sensitivity analysis demonstrated that the observed risk estimate was not robust, which changed from 0.17 (95\% CI $0.04-$ 0.29 ) to 0.57 (95\% CI 0.44-0.69; online suppl. Fig. S4C).

However, we found this association was statistically significant when analyzed categorically (ES 1.58, 95\% CI 1.22-2.06, $I^{2} 43.4 \%$; Fig. 4d). No publication bias existed in the meta-analysis ( $p=0.871$; online suppl. Fig. S3D). The sensitivity analysis also presented a robust result that was not influenced by individual studies (online suppl. Fig. S4D).

\section{Discussion}

This meta-analysis included 2,205 participants from 3 cross-sectional studies with VFA measurements, 12,429 participants from 11 cross-sectional studies with WC measurements, and 2,114 participants from 4 cross-sec-

Abdominal Obesity Correlates with DKD

More Closely tional studies, which demonstrated that WC, VFA and WHR/WHtR were associated with greater odds of DKD in type 2 diabetic patients. The results further supported abdominal obesity might play an important role in the pathophysiology of DKD in type 2 diabetic patients.

The relationship between abdominal obesity and DKD was reported by previous studies. In a cross-sectional research of 1,016 individuals with T2DM, patients with DKD had significantly higher VFA or WC levels than those without DKD [22]. Rossi et al. [25] found abdominal obesity, defined with WC, substantially increased the risk of microalbuminuria. However, some researchers reached the opposite conclusion. Man et al. [11] found that individuals with abdominal obesity had no association with DKD in T2DM (male: OR 1.45, 95\% CI 0.882.37; female: OR 1.69, 95\% 0.56-5.11). Kanakamani et al. [12] reported that WC was negatively associated with macroalbuminuria (OR $0.41,95 \%$ CI $0.22-0.57$ ) but not with microalbuminuria (OR 1.43, 95\% CI 0.83-2.47). Therefore, the results concerning this association should be investigated. In our study, we found that abdominal obesity parameters were significantly associated with increased odds of having DKD. However, high heterogeneity ( $\left.I^{2} 85.3 \%\right)$ was detected in our meta-analysis, which might be due to the difference in the definition of abdominal obesity. For instance, Asakawa et al. [17] defined abdominal obesity as VFA $\geq 100 \mathrm{~cm}^{2}$, Lu et al. [24] and Rossi et al. [25] identified abdominal obesity as $W C \geq 90 \mathrm{~cm}$ for men or $80 \mathrm{~cm}$ for women, while Tseng [10] defined abdominal obesity with WHR/WHtR. Hence, we assessed the relationship between different parameters of abdominal obesity and DKD in subgroup analysis.

In the WC subgroup analysis, patients with DKD had higher WC levels than those without DKD (SMD 0.17, 95\% CI 0.10-0.24), and this association became enhanced when we analyzed the dichotomized WC (ES 1.57, 95\% CI $1.32-1.86, p=0.039$ ). In the VFA subgroup, a tight association of continuous VFA with DKD in type 2 diabetic patients had been reported (SMD 0.27, 95\% CI 0.03$0.50, p=0.014)$. Additionally, in the WHR/WHtR subgroup, the categorized WHR/WHtR was significantly associated with an increase in the odds of DKD (ES 1.58, 95\% CI 1.22-2.06), while this association became attenuated when we analyzed the continuous WHR/WHtR (SMD 0.43, 95\% CI -0.12 to 0.97 ). In our meta-analysis, a positive correlation between abdominal obesity parameters (continuous VFA or WC) and DKD was confirmed, and type 2 diabetic patients with DKD were more likely to have abdominal obesity (categorized using WC or WHR/WHtR). 
Although the mechanisms about the relationship between abdominal obesity and DKD are still unclear, several hypotheses may be proposed. First, visceral fat can cause an increase in adipocytokines, such as free fatty acids, tumor necrosis factor- $\alpha$ and interleukin- 6 , which result in insulin resistance, mitochondrial dysfunction, oxidative stress and eventual renal damage [31,32]. Second, adipose tissue activates the renin-angiotensin system or the sympathetic nervous system, which allows for the changes in renal hemodynamics and sodium retention, leading to renal damage $[33,34]$. In addition, other manifestations of metabolic syndrome such as dyslipidemia, insulin resistance and hypertension also play an important role in the occurrence and development of DKD [35]. For instance, dyslipidemia can induce a series of pathophysiological changes such as endothelial dysfunction and oxidative stress, stimulate glomerular sclerosis, interstitial fibrosis and eventual renal dysfunction [36].

Our meta-analysis had some strengths. First, we used different parameters of abdominal obesity, including VFA, WC and WHR/WHtR, to evaluate the association of abdominal obesity with DKD, with more comprehensiveness and rationality. Second, the meta-analysis could avoid some potential errors through the subgroup analysis. The significant limitation in our meta-analysis is that all included studies were based on cross-sectional design, which could not confirm the causal association between abdominal obesity and DKD. Therefore, large prospective cohorts were needed to confirm the association of abdominal obesity with DKD in the future. For another, the adjustments for potential confounders differed in each study, and the influence of possible confounders on the meta-analysis could not be entirely excluded.

\section{Conclusion}

In summary, this meta-analysis demonstrated that abdominal obesity parameters (continuous VFA or WC) were associated with increased odds of DKD, and type 2 diabetic patients with DKD were more likely to have abdominal obesity (categorized using WC or WHR/WHtR). Large prospective cohort studies should be carried out to confirm the significant association or causality between abdominal obesity and DKD in the future.

\section{Acknowledgement}

The authors thank the First Affiliated Hospital of Chongqing Medical University.

\section{Statement of Ethics}

This study is a meta-analysis, and all analyses are based on previous published studies; thus, no ethical approval and patient consent are required.

\section{Conflict of Interest Statement}

The authors have no conflicts of interest in this work.

\section{Funding Sources}

No external funding was received for this work. Internal funding was provided by the National Natural Science Foundation of China (grant No. 81700754), the Technological Innovation and Application Development Project of Chongqing (grant No. cstc2019iscxmsxmX0207), the Chongqing Science and Health Joint Medical Research Project (grant No. 2018GDRC004), HighEnd Medical Talents of Middle-Aged and Young People in Chongqing (yuweiren [2015] 49) and the Bethune Merck diabetes research fund (grant No. G2018030).

\section{Author Contributions}

The first draft of the manuscript was written by Q.Z.; Q.Z., X.Y. and Z.W. designed the study, oversaw the data collection and performed data analysis. Z.W. contributed to the critical revisions of the article for important intellectual content. All authors took part in revising this meta-analysis, gave final approval of the version to be published and agree to be accountable for all aspects of the work.

\section{Data Availability Statement}

Full data sets can be obtained from the corresponding author at towzh713@126.com.

The authors confirm that the manuscript is an honest, accurate and transparent research; important aspects of the research have not been omitted; all discrepancies with the planned and registered research have been explained.
References
1 Molitch ME, Defronzo RA, Franz MJ, Keane WF, Mogensen CE, Parving HH, et al. American Diabetes Association: nephropathy in diabetes. Diabetes Care. 2004;20(11):117.

2 Barkoudah E, Skali H, Uno H, Solomon SD, Pfeffer MA. Mortality rates in trials of subjects with type 2 diabetes. J Am Heart Assoc. 2012 Feb;1(1):8-15.

3 Vazquez G, Duval S, Jacobs DR Jr, Silventoinen K. Comparison of body mass index, waist circumference, and waist/hip ratio in predicting incident diabetes: a meta-analysis. Epidemiol Rev. 2007;29(1):115-28. 
4 Nguyen NT, Nguyen XM, Lane J, Wang P. Relationship between obesity and diabetes in a US adult population: findings from the $\mathrm{Na}-$ tional Health and Nutrition Examination Survey, 1999-2006. Obes Surg. 2011 Mar;21(3):351-5.

5 Gross ML, Dikow R, Ritz E. Diabetic nephropathy: recent insights into the pathophysiology and the progression of diabetic nephropathy. Kidney Int. 2005 Apr;67(94):S50-3.

6 Pinto-Sietsma SJ, Navis G, Janssen WM, de Zeeuw D, Gans RO, de Jong PE; PREVEND Study Group. A central body fat distribution is related to renal function impairment, even in lean subjects. Am J Kidney Dis. 2003 Apr;41(4):733-41.

7 de Boer IH, Sibley SD, Kestenbaum B, Sampson JN, Young B, Cleary PA, et al.; Diabetes Control and Complications Trial/Epidemiology of Diabetes Interventions and Complications Study Research Group. Central obesity, incident microalbuminuria, and change in creatinine clearance in the epidemiology of diabetes interventions and complications study. J Am Soc Nephrol. 2007 Jan;18(1):23543.

8 Hanai K, Babazono T, Nyumura I, Toya K, Ohta M, Bouchi R, et al. Involvement of visceral fat in the pathogenesis of albuminuria in patients with type 2 diabetes with early stage of nephropathy. Clin Exp Nephrol. 2010 Apr;14(2):132-6.

9 Blaslov K, Bulum T, Duvnjak L. Waist-toheight ratio is independently associated with chronic kidney disease in overweight type 2 diabetic patients. Endocr Res. 2015;40(4):194-

10 Tseng $\mathrm{CH}$. Waist-to-height ratio is independently and better associated with urinary albumin excretion rate than waist circumference or waist-to-hip ratio in chinese adult type 2 diabetic women but not men. Diabetes Care. 2005 Sep;28(9):2249-51.

11 Man RE, Gan AT, Fenwick EK, Gupta P, Wong MY, Wong TY, et al. The Relationship between Generalized and Abdominal Obesity with Diabetic Kidney Disease in Type 2 Diabetes: A Multiethnic Asian Study and MetaAnalysis. Nutrients. 2018 Nov;10(11):1685.

12 Kanakamani J, Ammini AC, Gupta N, Dwivedi SN. Prevalence of microalbuminuria among patients with type 2 diabetes mellitus - a hospital-based study from north India. Diabetes Technol Ther. 2010 Feb;12(2):1616.

13 Grundy SM, Cleeman JI, Daniels SR, Donato KA, Eckel RH, Franklin BA, et al.; American
Heart Association; National Heart, Lung, and Blood Institute. Diagnosis and management of the metabolic syndrome: an American Heart Association/National Heart, Lung, and Blood Institute scientific statement. Circulation. 2005 Oct;112(17):2735-52.

14 World Health Organization. Physical status: the use and interpretation of anthropometry. Report of a WHO Expert Committee. Geneva: WHO; 1995. Tech Rep Ser No 854, p. $1-452$.

15 Eiji O. New criteria for "obesity disease." Circ J. 2006;70(1):150

16 Levey AS, Coresh J, Balk E, Kausz AT, Levin A, Steffes MW, et al.; National Kidney Foundation. National Kidney Foundation practice guidelines for chronic kidney disease: evaluation, classification, and stratification. Ann Intern Med. 2003 Jul;139(2):137-47.

17 Asakawa H, Tokunaga K, Kawakami F. Relationship of abdominal fat with metabolic disorders in diabetes mellitus patients. Diabetes Res Clin Pract. 2002 Feb;55(2):139-49.

18 O'Rourke K, Shea B, Wells GA. Meta-analysis of clinical trials. New York: Springer; 2001.

19 Higgins JP, Thompson SG. Quantifying heterogeneity in a meta-analysis. Stat Med. 2002 Jun;21(11):1539-58.

20 Egger M, Davey Smith G, Schneider M, Minder C. Bias in meta-analysis detected by a simple, graphical test. BMJ. 1997 Sep;315(7109):629-34.

21 Hoeppner MA. NCBI Bookshelf: books and documents in life sciences and health care. Nucleic Acids Res. 2013 Jan;41(Database issue):D1251-60.

$22 \mathrm{Hu}$ J, Yang S, Zhang A, Yang P, Cao X, Goswami R, et al. Associations between abdominal and general obesity and diabetic kidney disease: a cross-sectional study and a prospective study. Lancet Diab Endocrinol. 2016;17(11):S35

23 Chung MM, Fang SC, Tavintharan S, Keven A, Yee KP, Ming LSB, et al. Gain in adiposity over 3 years is associated with progressive renal decline in multi-ethnic South-East Asians with type 2 diabetes. J Diabetes. 2019 Apr;11(4):316-25.

24 Lu B, Yang Z, Wang M, Yang Z, Gong W, Yang $Y$, et al. High prevalence of diabetic neuropathy in population-based patients diagnosed with type 2 diabetes in the Shanghai downtown. Diabetes Res Clin Pract. 2010 Jun;88(3):289-94.

25 Rossi MC, Nicolucci A, Pellegrini F, Comaschi M, Ceriello A, Cucinotta D, et al. Identifying patients with type 2 diabetes at high risk of microalbuminuria: results of the $\mathrm{DE}$ -
MAND (Developing Education on Microalbuminuria for Awareness of reNal and cardiovascular risk in Diabetes) Study. Nephrol Dial Transplant. 2008 Apr;23(4):1278-84.

26 Chiang SC, Lee JK, Chen CH, Chuang LM, Tsan KW, Sheu WH, et al. Justifying the high prevalence of microalbuminuria for type 2 diabetic patients in Taiwan with conditional probability approach - a DEMAND II study. J Chin Med Assoc. 2011 Jan;74(1):3-10.

27 Cheng Y, Zhang H, Chen R, Yang F, Li W, Chen L, et al. Cardiometabolic risk profiles associated with chronic complications in overweight and obese type 2 diabetes patients in South China. PLoS One. 2014 Jul;9(7):e101289.

28 Chung HF, Al Mamun A, Huang MC, Long KZ, Huang YF, Shin SJ, et al. Obesity, weight change, and chronic kidney disease in patients with type 2 diabetes mellitus: a longitudinal study in Taiwan. J Diabetes. 2017 Nov;9(11):983-93.

29 Hanai K, Babazono T, Iwamoto Y. Renal manifestations of metabolic syndrome in type 2 diabetes. Diabetes Res Clin Pract. 2008 Feb;79(2):318-24.

30 Pasko N, Toti F, Strakosha A, Thengjilli E, Shehu A, Dedej T, et al. Prevalence of microalbuminuria and risk factor analysis in type 2 diabetes patients in Albania: the need for accurate and early diagnosis of diabetic nephropathy. Hippokratia. 2013 Oct;17(4):33741.

31 Hall ME, do Carmo JM, da Silva AA, Juncos LA, Wang Z, Hall JE. Obesity, hypertension, and chronic kidney disease. Int J Nephrol Renovasc Dis. 2014 Feb;7:75-88.

32 Eguchi M, Tsuchihashi K, Saitoh S, Odawara Y, Hirano T, Nakata T, et al. Visceral Obesity in Japanese Patients with Metabolic Syndrome: Reappraisal of Diagnostic Criteria by CT Scan. Hypertens Res. 2007 Apr;30(4):31523.

33 Hall JE, Henegar JR, Dwyer TM, Liu J, Da Silva $\mathrm{AA}$, Kuo JJ, et al. Is obesity a major cause of chronic kidney disease? Adv Ren Replace Ther. 2004 Jan;11(1):41-54.

34 Wahba IM, Mak RH. Obesity and obesity-initiated metabolic syndrome: mechanistic links to chronic kidney disease. Clin J Am Soc Nephrol. 2007 May;2(3):550-62.

35 Zhu Q, Liu C. Metabolic syndrome and Diabetic kidney disease. Int J Urol Nephrol. 2007;27(005):711-5.

36 Breyer MD, Böttinger E, Brosius FC 3rd, Coffman TM, Harris RC, Heilig CW, et al.; AMDCC. Mouse models of diabetic nephropathy. J Am Soc Nephrol. 2005 Jan;16(1):27-45. 